\title{
Simple and Sensitive Determination of Citrinin in Monascus by GC-Selected Ion Monitoring Mass Spectrometry
}

\author{
Pin-Yen SHU and Cheng-Huang LiN ${ }^{\dagger}$ \\ Department of Chemistry, National Taiwan Normal University, 88 Sec. 4, Tingchow Road, Taipei, Taiwan
}

\begin{abstract}
A new method for the qualitative and quantitative analysis of citrinin in Monascus by gas-chromatography-selected ion monitoring (SIM) mass spectrometry has been developed. GC separation of citrinin in Monascus extract was achieved without the need for chemical derivatization, and could be detected as a single peak when the SIM mode selected 5 prominent fragmentations $(\mathrm{m} / \mathrm{z}$ of $220,205,177,105$ and 91). The quantitative detection limit for citrinin was $\sim 1 \mathrm{ppb}$. Finally, the GC-separated analyte from Monascus extract, at a retention time of $10.89 \mathrm{~min}$, was examined by the method of pattern recognition by comparison with a citrinin standard. The results show that the 2 compounds had a $94 \%$ similarity when the SIM mode was used.
\end{abstract}

(Received September 21, 2001; Accepted November 28, 2001)

\section{Introduction}

Citrinin, a fungal metabolite, was first isolated from Penicillium citrinum in $1931^{1}$ and has been detected as a natural contaminant of wheat, rye, barley, oat and Monascus. In fact, the mycotoxic citrinin is a secondary metabolite, which is produced by several fungal species of the genera Penicillium and Aspergillus. This mycotoxin, which could be present either naturally or artificially, at enhanced levels, may cause a public health problem, ${ }^{2-5}$ such as the "yellowed rice" problem in Japan. Thus far, several TLC multi-mycotoxin methods have been proposed, but they exhibit relatively low sensitivities. ${ }^{6-8}$ Reversed-phase HPLC methods with either UV absorbance or fluorescence detection have also been applied..$^{9-11}$ At this time, GC/MS is currently a reliable method of detection in many fields. However, the majority of mycotoxins are not volatile and must be derivatized in order to make them amenable to GC. ${ }^{12}$ Even though several derivatization methods have been developed, this procedure is frequently time-consuming and is sometimes lacking in specificity. To overcome these limitations, a cold on-column injection technique coupled with GC/MS was developed for the direct analysis of metabolites produced by several Fusarium organisms without any chemical derivatization. ${ }^{13}$

In this study, we report on a new and highly sensitive method for the detection of citrinin in Monascus using GC-selected ion monitoring (SIM) mass spectrometry. This method, which does not require a derivatization procedure, was successfully applied to the analysis of citrinin in commercial Monascus products.

\section{Experimental}

Citrinin standard and reagents

Commercially pure citrinin [IUPAC: $(3 R, 4 S)-4,6$-dihydro-8hydroxy-3,4,5-trimethyl-6-oxo-3H-2-benzopyran-7-carboxylic

To whom correspondence should be addressed.

E-mail: chenglin@cc.ntnu.edu.tw acid] and chloroform $\left(99^{+} \%\right)$ were purchased from Sigma (St. Louis, MO, USA). Six commercial samples of Monascus red rice were acquired from various drugstores in Taipei, Taiwan.

\section{Apparatus}

A gas chromatograph (GC 5890 Hewlett-Packard, Avondale, PA, USA) equipped with a mass spectrometer (Hewlett-Packard 5973 mass selective detector) was used. A capillary column (30 $\mathrm{m} \times 0.25 \mathrm{~mm}$ i.d.) with a piece of HP-5MS (cross-linked 5\% PH ME siloxane) bonded stationary phase film $0.25 \mu \mathrm{m}$ thickness (Agilent Technologies, USA) was used. The inlet temperature was maintained at $200^{\circ} \mathrm{C}$. The column oven was held at $70^{\circ} \mathrm{C}$ for $3 \mathrm{~min}$, then programmed from $70-280^{\circ} \mathrm{C}$ at $15^{\circ} \mathrm{C} / \mathrm{min}$ and, finally, held for $5 \mathrm{~min}$. Helium carrier gas was used at a constant flow-rate of $1 \mathrm{~mL} / \mathrm{min}$ without splitting mode. Data were collected using the Hewlett-Packard Chem-Station software. The mass spectrometric conditions were as follows: SIM mode; ionization energy, $12 \mathrm{eV}$; ion source temperature, $230^{\circ} \mathrm{C}$. The mass selective detector was operated in the SIM mode at a scan rate of 1.25 scans per second.

\section{Liquid-liquid extraction procedure}

Monascus samples were finely ground and $2 \mathrm{~g}$ of the resulting powder was extracted with $2 \mathrm{~mL}$ of chloroform. After $15 \mathrm{~min}$ of sonication and a 5 min centrifugation at $5000 \mathrm{rpm}$ at room temperature, the upper layer was filtered through a $0.45 \mu \mathrm{m}$ syringe filter and then degassed for $5 \mathrm{~min}$. For each experiment, a $1.0 \mu \mathrm{L}$ aliquot of the chloroform solution were injected onto the GC/MS.

\section{Results and Discussion}

\section{GC-SIM analysis for citrinin}

The total ion chromatogram (TIC) of the citrinin standard is shown in Fig. 1. When analyzed by GC/MS in the electron impact (EI) mode, the detected peaks with retention times of 12.32 and $15.50 \mathrm{~min}$ did not provide for a specific characterization of citrinin, as shown in Fig. 1 inset (a) and (b). In fact, all of the elutes along the time axis were derived from 


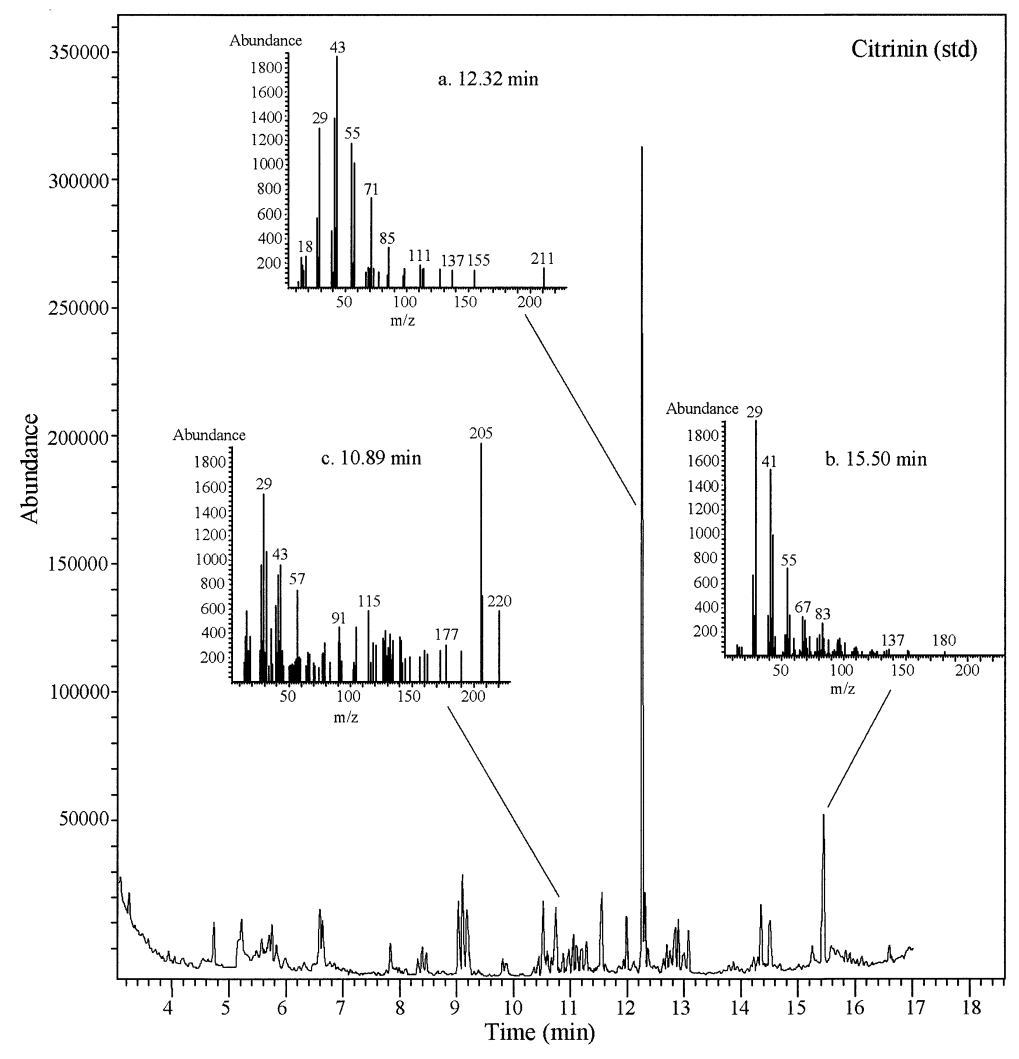

Fig. 1 Total ion chromatograms of a citrinin standard. Insets (a), (b) and (c) are corresponding to mass fragmentation spectra detected at $12.32,15.50$ and $10.89 \mathrm{~min}$.

citrinin, but most of them did not reveal sufficient information about the parent ion of citrinin. They were produced either by thermal decomposition during the separation process or by electron impact during the detection process. This is probably the reason why there are no reports on the direct analysis of citrinin without derivatization, which is required for most mycotoxins. ${ }^{13}$ However, we found that the peak with a retention time of 10.89 min represents a potentially useful location for the direct identification of citrinin because of some specific fragmentations, such as $\mathrm{m} / \mathrm{z}$ of 220 and 205, etc., as shown in inset (c) of Fig. 1. In order to understand and characterize these peaks, we used the commercially available computer software Mass Frontier 1.0, which was obtained from HighChem, Ltd. (http://www.highchem.com, Slovakia), to perform some calculations and predictions. All of the possible fragmentation mass spectra were automatically predicted and suggested by this program when the chemical structure of citrinin was entered (in EI mode). Based on the computer calculation, numerous situations were found to be possible. As a result, we selected 5 prominent fragmentation peaks $(\mathrm{m} / \mathrm{z}$ of $220,205,177,105$ and 91) as markers. A portion of the predicted fragmentation pattern of citrinin is shown in Fig. 2. Herein, when analyzed by SIM mode using these 5 prominent ions, a single peak with a retention time of $10.89 \mathrm{~min}$ appeared, as shown in Fig. 3. It is obvious that this method is able to efficiently simplify the chromatogram and provide a single peak for identification. Herein, the concentration of citrinin was $10 \mathrm{ppm}$. Other series of different $\mathrm{m} / \mathrm{z}$ values, e.g. $\mathrm{m} / \mathrm{z}=220,205,189,177,161,149$, 105 and 91 (based on computer suggestions), were also selected and the results showed that the same peak was detected, even though the height of the peak was not exactly the same. Figure 4 illustrates the selected ion current profile for citrinin standards over a concentration range $1-10 \mathrm{ppb}$. The detection limit was $0.6 \mathrm{ppb}(S / N=3)$. The GC-SIM technique clearly provides a simple and sensitive method for the determination of citrinin. The inset in Fig. 4 shows the relationship of the inlet temperature of the $\mathrm{GC}$ injection port and the relative ion currents. We found that the inlet temperature, when maintained at $200^{\circ} \mathrm{C}$, provided better detection limits of citrinin. Therefore, in all of the following experiments, the inlet temperature was set at $200^{\circ} \mathrm{C}$.

\section{Analysis of actual samples}

Figure 5(A) shows the total ion chromatogram profile for the Monascus extract of the drugstore I product. We found a tiny peak with a retention time of $10.89 \mathrm{~min}$. With the SIM profile, this peak could be simplified, as shown in Fig. 5(B). The inset shows the corresponding mass fragmentation spectrum. Based on the calibration curve in the range of $1-100 \mathrm{ppm}$, the concentration of citrinin in this Monascus was determined to be $25.1 \mathrm{ppm}$. We also found that the concentration of citrinin in Monascus was dramatically decreased after boiling in water $\left(\sim 100^{\circ} \mathrm{C}\right)$, as shown in the Fig. 5(A) inset. After about 20-min of heating, the concentration of citrinin can be decreased by $50 \%$. Table 1 shows a summary of 6 Monascus samples which were randomly purchased from different drugstores in Taipei. Citrinin can be detected in all of the 6 commercial Monascus samples at concentrations varying between 4.2 to $25.1 \mathrm{ppm}$. Citrinin is known to be a hepato-nephrotoxin in a wide range of species, even though reports on its mutagenicity and genotoxicity were controversial and, in short-term mutagenicity tests, citrinin was found to be negative. ${ }^{14-16}$ 
<smiles>C=CC1=C(C)C(=O)C(=[O+]C=C2C(=O)C(C(=O)O)C(=O)C(C)=C2C=C)C(=O)C1=C</smiles>

Fig. 2 Fragmentation pattern of citrinin obtained by Mass Frontier 1.0 .

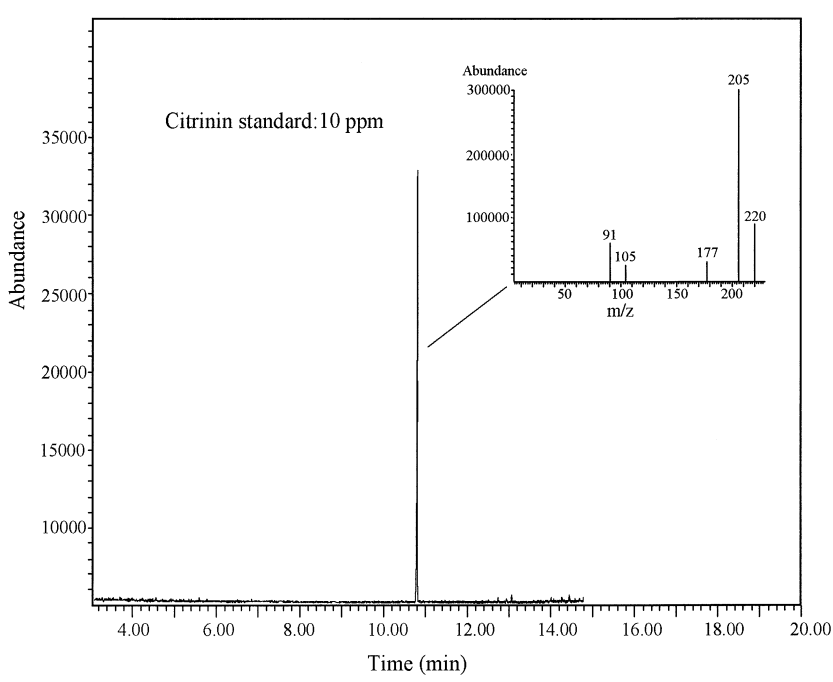

Fig. 3 Selected-ion monitoring (SIM) chromatogram of a citrinin standard $(\mathrm{m} / \mathrm{z}$ of $220,205,177,105$ and 91$)$. The inset corresponds to the mass fragmentation spectrum.

\section{Precision: MS analysis by pattern recognition}

This section describes an approach involving the recognition of patterns of mass spectral lines, which are produced as a result of GC-separated analytes. The pattern recognition provides a method for the prediction of chemical structure and can be applied to samples that have not previously been examined. ${ }^{17}$ Indeed, when a complete mass database is available, a sample molecule can be accurately identified using the mass spectral data accumulated for a series of authentic compounds. However, without the derivation procedure, a mass spectrum of citrinin has not been reported by GC/MS. Herein, we used the method of pattern recognition to describe the similarity of two $\mathrm{GC}$ elutes, both of which had the same GC retention times. In order to correlate the mass spectral features of two different GC-separated analytes, a cross correlation factor $(\mathrm{CCF})$ is

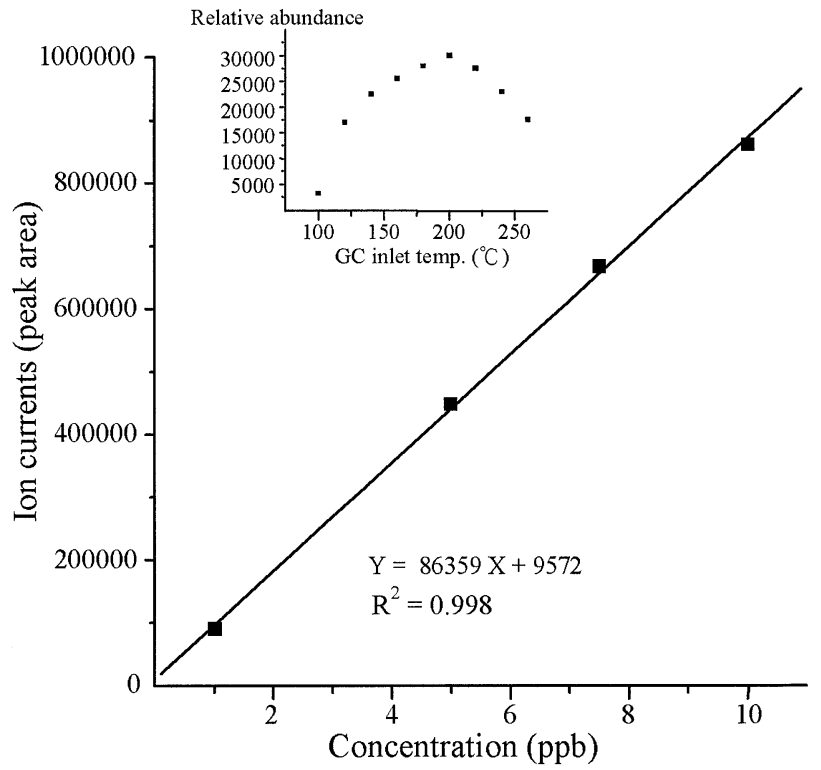

Fig. 4 Calibration graph of citrinin: linear regression of the concentration and peak area. Inset, the relationship of ion currents and temperatures of the GC inlet.

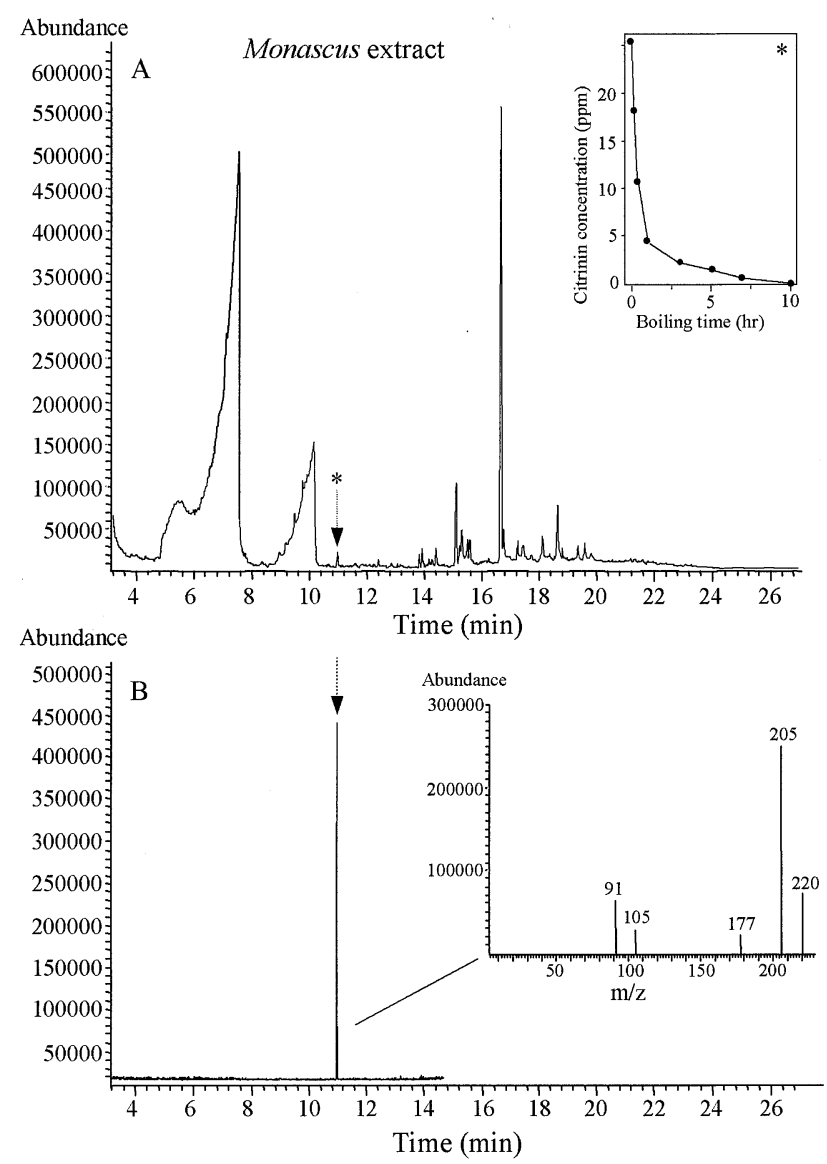

Fig. 5 (A) Total ion chromatograms of a Monascus extract. Inset, the concentration of citrinin in Monascus was decreased after boiling in water $\left(\sim 100^{\circ} \mathrm{C}\right)$. (B) $\operatorname{SIM}(\mathrm{m} / \mathrm{z}$ of $220,205,177,105$ and 91) chromatogram of Monascus extract.

calculated between the mass spectra obtained from the GCseparated citrinin standard and the Monascus extract, both at 
A
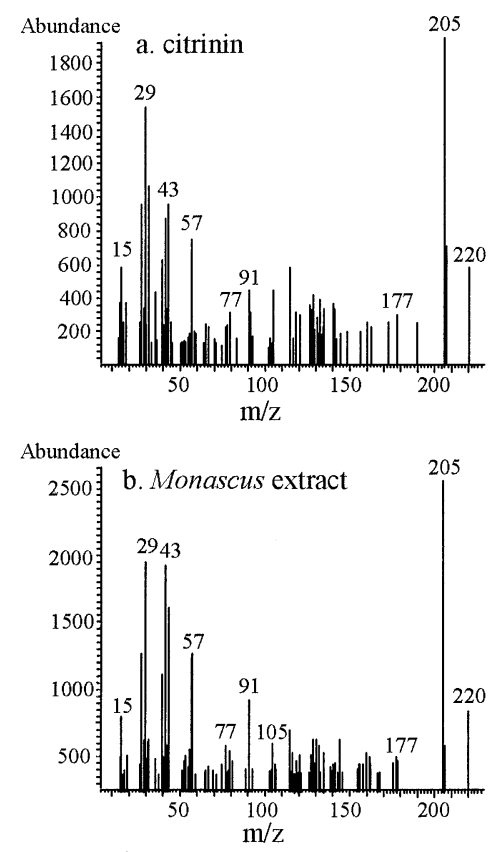

B

$\operatorname{SIM}(10.89 \mathrm{~min})$
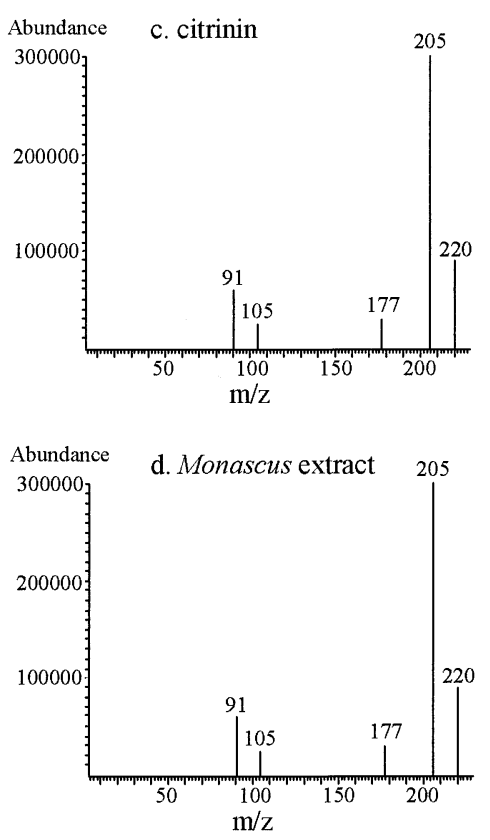

Fig. 6 (A) Mass fragmentation spectrum of total ion chromatograms. Spectrum a, citrinin standard; b, Monascus extract. (B) Mass fragmentation spectrum of SIM chromatograms. Spectrum a, citrinin standard; b, Monascus extract.

migration times of 10.89 min. The CCF value is calculated by

$$
\mathrm{CCF}=\frac{\sum_{n=1}^{n} A_{n} \times\left(1-D_{n}\right)}{\sum_{n=1}^{n} A_{n}}
$$

where $A_{n}$ is relative strength of the mass spectra and $D_{n}$ the deviance. If the CCF value is close to unity when the mass spectral feature of the standard is similar to that of the reference; $\mathrm{CCF}=1$ when $\mathrm{CCF}$ is calculated between two of the same molecules. In contrast, the $\mathrm{CCF}$ value is close to zero when the spectral features are completely different from each other. The CCF value was calculated using Microsoft-Excel on a personal computer. The spectral data used were the values of $\mathrm{m} / \mathrm{z}$ and their related intensities. In Fig. 6, frame (A) shows mass spectra of a citrinin standard (spectrum a) and a Monascus extract (spectrum b) detected at $10.98 \mathrm{~min}$; frame (B) shows the same GC-separated analytes detected by the SIM modes $(\mathrm{m} / \mathrm{z}$ of $220,205,177,105$ and 91). As a result, the values of the CCF of frames (A) and (B) were 0.84 and 0.94 , respectively. In the other words, the similarity of the TIC mode was $84 \%$, whereas the similarity was increased to $94 \%$ when the SIM mode was selected. Therefore, we assigned the peak detected at $10.89 \mathrm{~min}$ in Monascus extracts to citrinin.

\section{Conclusions}

We demonstrated a new method for the qualitative and quantitative analysis of citrinin in Monascus by gaschromatography-selected ion monitoring (SIM) mass spectrometry. Using this method, chemical derivatization is not necessary. The chromatographic separation of the citrinin in the
Table 1 Amounts (ppm) of citrinin detected in 6 different commercial samples of Monascus obtained from drugstores (in Taipei)

\begin{tabular}{lcr}
\hline Sample & Citrinin detected, ppm & \multicolumn{1}{c}{ CV } \\
\hline No. I & $25.1 \pm 2.5$ & $10.0 \%$ \\
No. II & $12.3 \pm 1.3$ & $10.5 \%$ \\
No. III & $8.7 \pm 0.6$ & $6.4 \%$ \\
No. IV & $5.1 \pm 1.0$ & $19.8 \%$ \\
No. V & $4.2 \pm 0.1$ & $2.2 \%$ \\
No. VI & $10.5 \pm 2.6$ & $24.3 \%$ \\
\hline
\end{tabular}

Monascus extract was achieved as a single peak when 5 prominent fragmentations $(\mathrm{m} / \mathrm{z}$ of $220,205,177,105$ and 91) were selected as markers. The detection limit of citrinin was $\sim 1$ ppb. The GC-separated analyte from Monascus extract was examined by pattern recognition. The similarity of the citrinin standard and the GC-separated analyte of Monascus extract was $94 \%$.

\section{Acknowledgements}

The authors would like to thank Dr. Yu-Ju Chen and Miss ShinI Tang (Institute of Chemistry, Academia Sinica, Taiwan) for the calculations of Mass Frontier 1.0 and for helpful discussions.

\section{References}

1. A. C. Hetherington and H. Raistrick, Trans. Roy. Soc. 
London, Ser. B, 1931, 220, 269.

2. L. B. Bullerman, J. Food Protect., 1979, 42, 65.

3. Ch. F. Jelinek, A. E. Pohland, and G. E. Wood, J. Assoc. Off. Anal. Chem., 1989, 72, 223.

4. P. B. Mislivec, J. Food Protect., 1981, 9, 723.

5. T. Taira and S. Yamatodani, Penishirin, 1947, 1, 275.

6. V. Betina, J. Chromatogr., 1989, 477, 187.

7. F. S. Chu, J. Anim. Sci., 1992, 70, 3950.

8. P. M. Scott, Tr. Anal. Chem., 1993, 12, 373.

9. D. Abramson, E. Usleber, and E. Martlbauer, J. AOAC. Int., 1999, 82, 1353.

10. J. C. Frisvad, J. Chromatogr., 1987, 392, 333.
11. B. I. Vazquez, C. Fente, C. Franco, A. Cepeda, P. Prognon, and G. Mahuzier, J. Chromatogr. A, 1996, 727, 185.

12. P. M. Scott, Food Addit. Contam., 1995, 12, 395.

13. Y. Onji, Y. Aoki, N. Tani, K. Umebayashi, Y. Kitada, and Y. Dohiu, J. Chromatogr. A, 1998, 815, 59.

14. M. H. Kuczuk, P. M. Benson, H. Heath, and A. W. Hayes, Mutat. Res., 1978, 53, 11.

15. F. E. Wuergler, U. Friedrich, and J. Schlatter, Mutat. Res., 1991, 261, 209.

16. M. Sabater-Vilar, R. F. M. Maas, and J. Fink-Gremmels, Mutat. Res., 1999, 4444, 7.

17. C.-H. Lin and T. Imasaka, Talanta, 1995, 42, 1111. 